\title{
Serum lipid profiles in patients with acute myocardial infarction in Hakka population in southern China
}

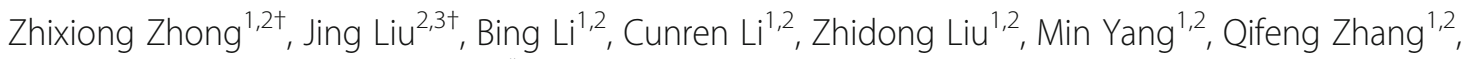
Wei Zhong ${ }^{1,2}$ and Pingsen Zhao ${ }^{2,3^{*}}$

\section{Abstract}

Background: Little is known about serum lipid levels comparison of patients with acute myocardial infarction (AMI) in Hakka patients in southern China. To estimate the prevalence lipid profiles in Hakka patients with AMI in southern China.

Method: We analyzed 1382 patients with a first AMl in Hakka patients in southern China between Jan. 2015 and Dec. 2015. Results: Our findings demonstrated that low-density lipoprotein cholesterol (LDL), total cholesterol (TC), and triglyceride (TG) were higher in nonelderly than in elderly for males. There were significant differences in TC, LDL, HDL, and TG among various age groups for both males and female patients $(P<.05)$. TC, LDL, HDL, and TG were higher in females than males for the elderly, and the LDL levels of females were higher in 70-79,80-89 year age groups than males. The HDL level of female patients was higher than males in those 50-59, 60-69, and 70-79 year age groups. Compared with males, females had higher level of TG in the 60-69, 70-79, and 80-89 year age groups and had higher level of TC in the 50-59, 70-79, and 80-89 year age groups, respectively. Isolated high TG (normal LDL + normal HDL+ high TG) was most common type of combined dyslipidemia for female elderly (22.2\%), female nonelderly (23.2\%) and male elderly (24.1\%) patients.

Conclusion: Our results confirmed that serum lipid levels varied in age and gender in Hakka patients with acute myocardial infarction. Dyslipidemia is more prevalent in the non-elderly than in the elderly for males. Levels of TC, LDL, HDL, and TG were higher in females than males for the elderly Hakka population in southern China.

Keywords: Dyslipidemia, Coronary heart disease, Myocardial infarction, Low-density lipoprotein cholesterol, High-density lipoprotein cholesterol, Total cholesterol, Cholesterol, Triglyceride

\footnotetext{
* Correspondence: zhaopingsen01@163.com; zhaopingsen@hotmail.com

${ }^{\dagger}$ Equal contributors

${ }^{2}$ Clinical Core Laboratory, Meizhou People's Hospital (Huangtang Hospital),

Meizhou Hospital Affiliated to Sun Yat-sen University, Meizhou 514031,

People's Republic of China

${ }^{3}$ Center for Precision Medicine, Meizhou People's Hospital (Huangtang

Hospital), Meizhou Hospital Affiliated to Sun Yat-sen University, Meizhou

514031, People's Republic of China

Full list of author information is available at the end of the article
} 


\section{Background}

In recent years, cardiovascular disease (CVD) is the leading cause of mortality and morbidity worldwide in both male and female populations, that is widely accepted [1, 2]. Some clinical studies show that the incidence of coronary artery disease (CAD) continue to increase; about 1 in 5 Chinese is suffering from CAD [2].Cardiovascular disease is regarded as a multifactorial disease, which affected by the environment and genetic factors. Traditional cardiovascular risk factors, such as smoking, drinking, diabetes, dyslipidemia and advanced age, can increase the risk of cardiovascular disease. [3] Among the many cardiovascular disease risk factors, dyslipidemia is considered as the most important factor, which is a strong predictor for cardiovascular outcomes after AMI. Lipid profile evaluation including total cholesterol (TC), triglycerides (TG), low-density lipoprotein (LDL-C) and high-density lipoprotein (HDL-C) allows an assessment of CVD risk. A growing evidences indicates that elevated concentrations of TG, TC, LDL-C and decreased HDL-C accelerate the development of atherosclerotic plaques [4-7].

Dyslipidemia is an important risk factor for cardiovascular disease after acute myocardial infarction. Different age and gender have different lipid levels. Previous studies have shown age- and gender- differences in lipid profile in AMI patients in East China, and dyslipidemia is biased toward young people, at the same time, some researchers also reported the lipid metabolism in urban and rural areas of southern China [8].However, little is known about age and gender-related lipid levels comparison of patients with acute myocardial infarction (AMI) in the Hakka population of southern China. Understanding age-and gender-related differences in post-AMI lipid profiles and the characteristic of dyslipidemia in the Hakka population in southern China may provide important implications for treatment and guide strategy to reduce age- and gender-related differences in outcomes.

\section{Methods \\ Subjects}

This is a retrospective study. A total of 1382 patients with first myocardial infarction were collected through Jan. 2015 and Dec. 2015 in Meizhou People's Hospital (Huangtang Hospital), Meizhou Hospital Affiliated to Sun Yat-sen University, China. The patients were excluded containing: (1) previous AMI; (2) Incomplete datas of lipid files; (3) Patients with chronic liver dysfunction, malignant tumors and other serious medical diseases; (4) Patients who were taking medications of lipid-lowering, such as statins and fibrates. The study approved by Human Ethics Committees of Meizhou People's Hospital (Huangtang Hospital), Meizhou Hospital Affiliated to Sun Yat-sen University,
Guangdong province, China. All the patients had signed the informed consent.

\section{Data collection and analysis}

Data were prospectively collected, such as gender, age and diabetes, obesity, hypertension and family history. Peripheral venous blood samples were collected within $24 \mathrm{~h}$ after admission for measuring lipid profiles. Lipid profiles were tested by selective solubilization method (AU5400 analyzer, Beckman Coulter, CA, USA).The continuous variables and categorical variables were expressed as mean \pm standard deviation, as $\%$, or frequencies with percentage, respectively. IBM SPSS Statistics 21.0 (IBM, Armonk, NY, USA) was used for all statistical analyses.

Differences lipid profiles between groups based on were analyzed using $X^{2}$ test, Kruskal-Wallis test or One-way ANOVA analysis of variance test. A probability value of $P$ $<0.05$ was considered significant for this study.

\section{Results}

Clinical characteristics of Hakka patients with AMI

The flow chart of study are show in Fig. 1.Sample clinical baseline characteristics of the AMI patients were shown in Table 1. There were 1382 patients with AMI were enrolled in this study. The average age were similar between nonelderly and elderly. (55 years for men in nonelderly and 75 years for men in elderly, 57 years for women in nonelderly and 77 years for women in elderly). The rate of smoking and Hypertension were significantly difference between nonelderly and elderly for males.

Levels of TC, LDL, and TG were lower in elderly than in non-elderly for the males. Female of lipid profiles, which containing TC, LDL, HDL, and TG, were all significantly higher females than in males for the elderly. However, in the non-elderly group, there were no

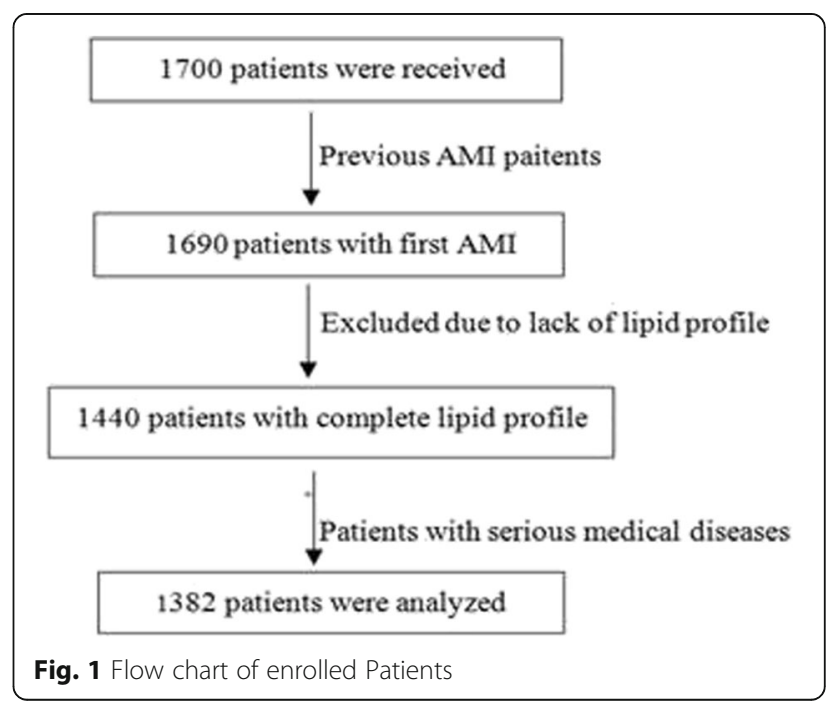


Table 1 Clinical characteristics of Hakka patients with AMI

\begin{tabular}{|c|c|c|c|c|}
\hline & \multicolumn{2}{|l|}{ Nonelderly } & \multicolumn{2}{|l|}{ Elderly } \\
\hline & Male & Female & Male & Female \\
\hline Cases & 485 & 86 & 586 & 225 \\
\hline Age (y) & $55 \pm 7$ & $57 \pm 6$ & $75 \pm 6$ & $77 \pm 7$ \\
\hline $\mathrm{BMI}$ & $22.4 \pm 1.5$ & $22.1 \pm 2.1$ & $22.4 \pm 2.2$ & $21.8 \pm 1.7$ \\
\hline Smoking, n (\%) & 251(51.8) & $0(0)^{* *}$ & $168(34.6)^{\# \#}$ & - \\
\hline Alcoholdrinking, n (\%) & $13(2.7)$ & $0(0)^{* *}$ & $26(4.4)$ & - \\
\hline Hypertension, n (\%) & 218(44.9) & $52(60) * *$ & $319(54.4)^{\# \#}$ & $132(22.5)^{* * \# \#}$ \\
\hline Diabetes, n (\%) & 137(28.2) & $37(43)^{* *}$ & 151(25.8) & $71(31.5)^{\#}$ \\
\hline CHD, n (\%) & $46(9.4)$ & $8(9.3)$ & $82(14)$ & $28(12.4)$ \\
\hline CVD, n (\%) & $27(5.5)$ & $10(11.6) *$ & $62(10.6)^{\# \#}$ & $24(10.7)$ \\
\hline $\mathrm{TC}(\mathrm{mmol} / \mathrm{L})$ & $4.88 \pm 1.21$ & $5.27 \pm 1.72$ & $4.58+1.15^{\# \#}$ & $5.16 \pm 1.67^{* *}$ \\
\hline $\mathrm{HDL}(\mathrm{mmol} / \mathrm{L})$ & $1.25 \pm 0.39$ & $1.47 \pm 0.399^{* *}$ & $1.3 \pm 0.4$ & $1.43 \pm 0.41^{* *}$ \\
\hline LDL (mmol/L) & $2.80 \pm 0.83$ & $2.83 \pm 0.92$ & $2.6 \pm 0.84^{\# \#}$ & $2.82 \pm 0.97^{* *}$ \\
\hline $\mathrm{TG}(\mathrm{mmol} / \mathrm{L})$ & $2.15 \pm 1.6$ & $2 \pm 1.33$ & $1.49 \pm 1^{\# \#}$ & $1.87 \pm 1.44^{* *}$ \\
\hline $\mathrm{TC} / \mathrm{HDL}$ & $4.06 \pm 1.04$ & $3.68 \pm 1.06^{* *}$ & $3.71 \pm 1.01^{\# \#}$ & $3.75 \pm 1.11$ \\
\hline LDL/HDL & $2.38 \pm 0.86$ & $2.01 \pm 1.72^{* *}$ & $2.14 \pm 0.83^{\# \#}$ & $2.08 \pm 0.84$ \\
\hline $\mathrm{TG} / \mathrm{HDL}$ & $1.78 \pm 1.18$ & $1.48 \pm 1.01^{*}$ & $1.27 \pm 1.10^{\# \#}$ & $1.38 \pm 0.98$ \\
\hline
\end{tabular}

AMI acute myocardial infarction, CHD coronary heart disease, CVD cerebrovascular disease, HDL high-density lipoprotein cholesterol, LDL low-density lipoprotein cholesterol, TC total cholesterol, TG triglyceride

Compared with males, ${ }^{*} P<.05$ and ${ }^{* * P}<.01$ for both the nonelderly and the elderly

Compared with the nonelderly, ${ }^{\#} P<.05$ and ${ }^{\# \#} P<.01$ for both males and females

significant differences in TC, LDL, TG between male and female except HDL.

\section{Age and gender differences of LDL levels in Hakka patients with AMI}

The levels of LDL in different age groups were statistically significant $(P<.05$ or $<.01$, Fig. $2 \mathrm{a})$ and females have higher LDL in both 70-79 (males vs. females, 2.61 \pm 0.90 vs. $2.86 \pm 0.87, P<.05$ ) and $80-89$ (males vs females, $2.46 \pm 0.70$ vs. $2.82 \pm 0.70, P<.05)$ year age groups compare to males (Fig. 2b). For male patients, LDL levels in $<40$ year age group was higher than those in 50-89 year age groups. At the same time, 40-49 and 50-59 year age groups were higher LDL levels compare to 70-79 and 80-89 year age groups (Fig. 2c). However, 50-59 year age group of LDL levels was higher than 4049 year age group for female patients (Fig. 2d).

\section{Age and gender differences of HDL levels in Hakka patients with AMI}

The levels of HDL in different age groups were statistically significant $(P<.05$ or $<.01$, Fig. 3a). The HDL levels of females in 50-59, 60-69, and 70-79 year age groups were higher than in males, respectively (Fig. 3b).Variance analysis showed that HDL levels in 80-89 year age group was higher than those $\leq 79$ year-old year age groups for male patients (Fig. 3c). Similarly, The data implicated HDL level only in 80-89 year age group was lower compare to 50-59 year age group for females patients (Fig. 3d).

\section{Age and gender differences of TG levels in Hakka patients with AMI}

The levels of TG among various age groups were statistically significant for males and females AMI patients $(P$ $<.05$ or <.01, Fig. 4a). Compared with males, females had higher level of TG in the 60-69, 70-79, and 80-89 year age groups respectively (Fig. 4b).Cross-comparison of arbitrary two age groups demonstrated 40-49 and50-59 year age groups were higher TG levels compare to in 60-69, $70-79,80-89$, and $\geq 90$ year age groups, as well as $60-$ 69 year age group was higher TG level compare with 7079, 80-89 year age groups for males (Fig. 4c). Pair wise comparison implicated TG level only in 70-79 year age group was lower than that in 50-59 year age group for females AMI patients (Fig. 4d).

\section{Age and gender differences of TC levels in Hakka patients with AMI}

The levels of TC among various age groups were statistically significant for males and females AMI patients $(P$ $<.05$ or $<.01$, Fig. 5a). The TC levels of females in the 50 $59,70-79$, and 80-89 year age groups were higher compare to males, respectively (Fig. 5b). Cross-comparison of arbitrary two age groups demonstrated 40-49 and50- 

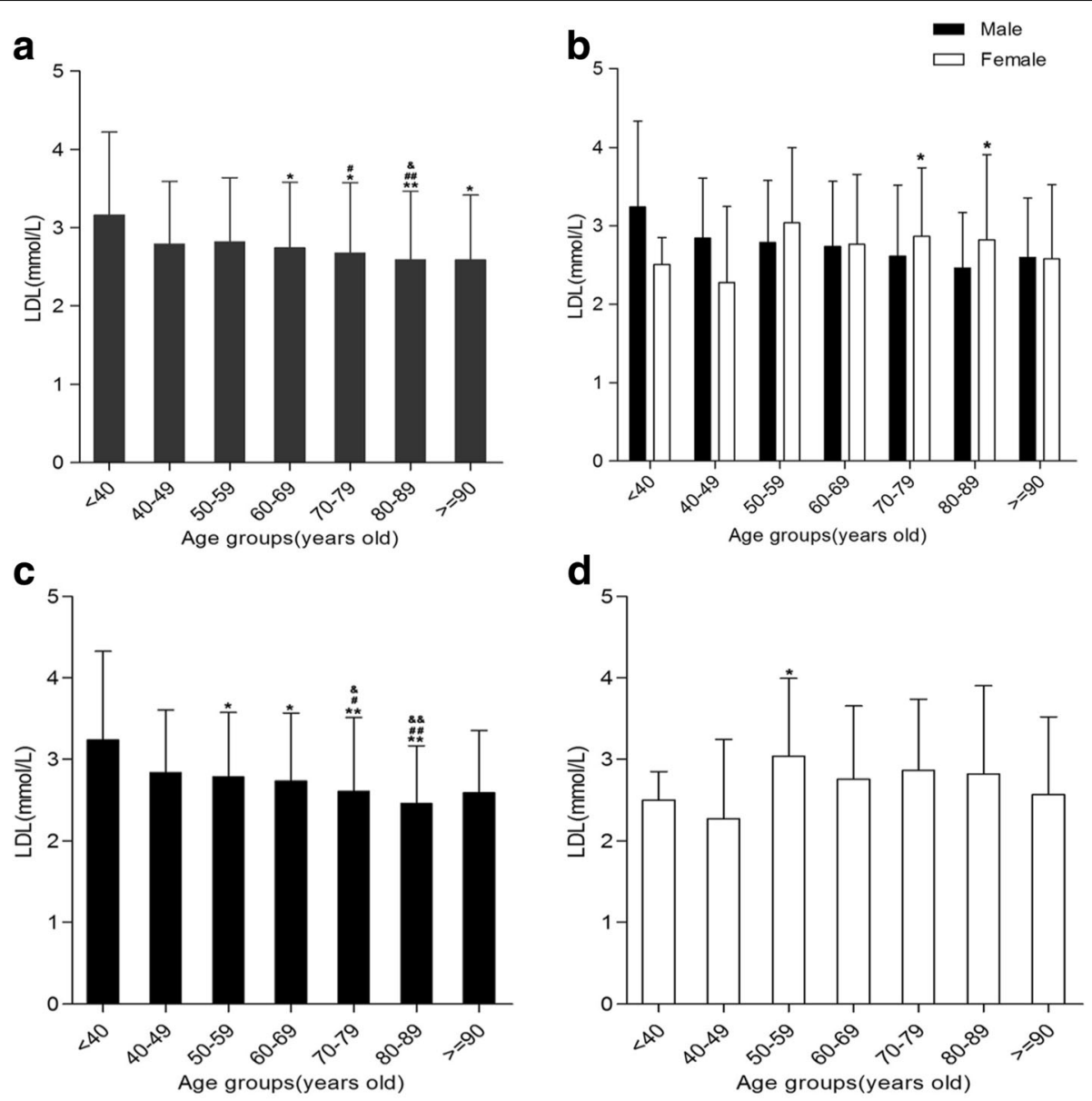

Fig. 2 Low-density lipoprotein (LDL) levels in various age groups. a Comparison between $<40$ years of age group and more than 60 years of various age groups, respectively, ${ }^{*} P<.05,{ }^{* *} P<0.01$. Comparison between 50 and 59 year age groups and $70-79$ and $80-89$ year age groups, respectively, ${ }^{\#} P<.05,{ }^{\# \#} P<.01$. Comparison between 60 and 69 year age groups and $80-89$ year age groups were made, ${ }^{\circledR} P<.05$. b Compared with males in the same age group, ${ }^{*} P<.05$. c For males, comparison between $<40$ years of age group and $50-89$ years of various age groups, respectively, ${ }^{*} P<.05,{ }^{*} P<.01$. Comparison between 40 and 49 and $50-59$ year age groups and $70-79$ and $80-89$ year age groups, respectively, ${ }^{\#} P<.05,{ }^{\# \#} P<.01 .^{\&} P<.05,{ }^{\& \&} P<.01$. d For females, Comparison between 40 and 49 and $50-59$ year age group, ${ }^{*} P<.05$

59 year age groups were higher TC levels compare to 70 $79,80-89$ age groups, respectively, and 60-69 year age group was higher TC level compare with 70-79 year age groups for males (Fig. 5c). Pair wise comparison implicated TG level in 50-59 year age group was higher than those in 40-49, 60-69,80-89 year age groups, respectively, for females AMI patients (Fig. 5d).

\section{Comparison of lipid abnormalities in elderly and non-elderly Hakka patients with AMI}

According to the Guideline for the Prevention and Treatment of Chinese Adult Dyslipidemia 2007, serum level of $\mathrm{HDL}<1.04 \mathrm{mmol} / \mathrm{L}(40 \mathrm{mg} / \mathrm{dL})$ and $\geq 1.04 \mathrm{mmol} / \mathrm{L}$ (40 mg/dL) were regarded as low and normal, respectively. Serum level of LDL $<3.37 \mathrm{mmol} / \mathrm{L}(130 \mathrm{mg} / \mathrm{dL})$ and $\geq 3.37 \mathrm{mmol} / \mathrm{L}(130 \mathrm{mg} / \mathrm{dL})$ were regarded as high and normal, respectively. Serum level of TC $<5.18 \mathrm{mmol} / \mathrm{L}$
$(200 \mathrm{mg} / \mathrm{dL})$ and $\geq 5.18 \mathrm{mmol} / \mathrm{L}(200 \mathrm{mg} / \mathrm{dL})$ were regarded as normal and high, respectively.; serum level of TG $<1.70 \mathrm{mmol} / \mathrm{L}(150 \mathrm{mg} / \mathrm{dL})$ and $\mathrm{TG} \geq 1.70 \mathrm{mmol} / \mathrm{L}$ $(150 \mathrm{mg} / \mathrm{dL})$ were regarded as normal and high, respectively [9]. The distribution of dyslipidemia in AMI patients were shown in Table 2. The results indicated the most common type of combined dyslipidemia was isolated high TG (normal LDL+ normal HDL+ high TG) that reflect in female elderly $(22.2 \%)$, female nonelderly $(23.2 \%)$ and male elderly (24.1\%). For males, the proportion of isolated high TG was higher in the non-elderly. In addition High LDL+ normal HDL + high TG, Categories of combined dyslipidemia were not statistically significant between the nonelderly and the elderly for females. Compare with the non-elderly females, males had lower the proportions of high LDL+ normal HDL + high TG is lower and higher the proportion of normal LDL+ low HDL+ high TG is 

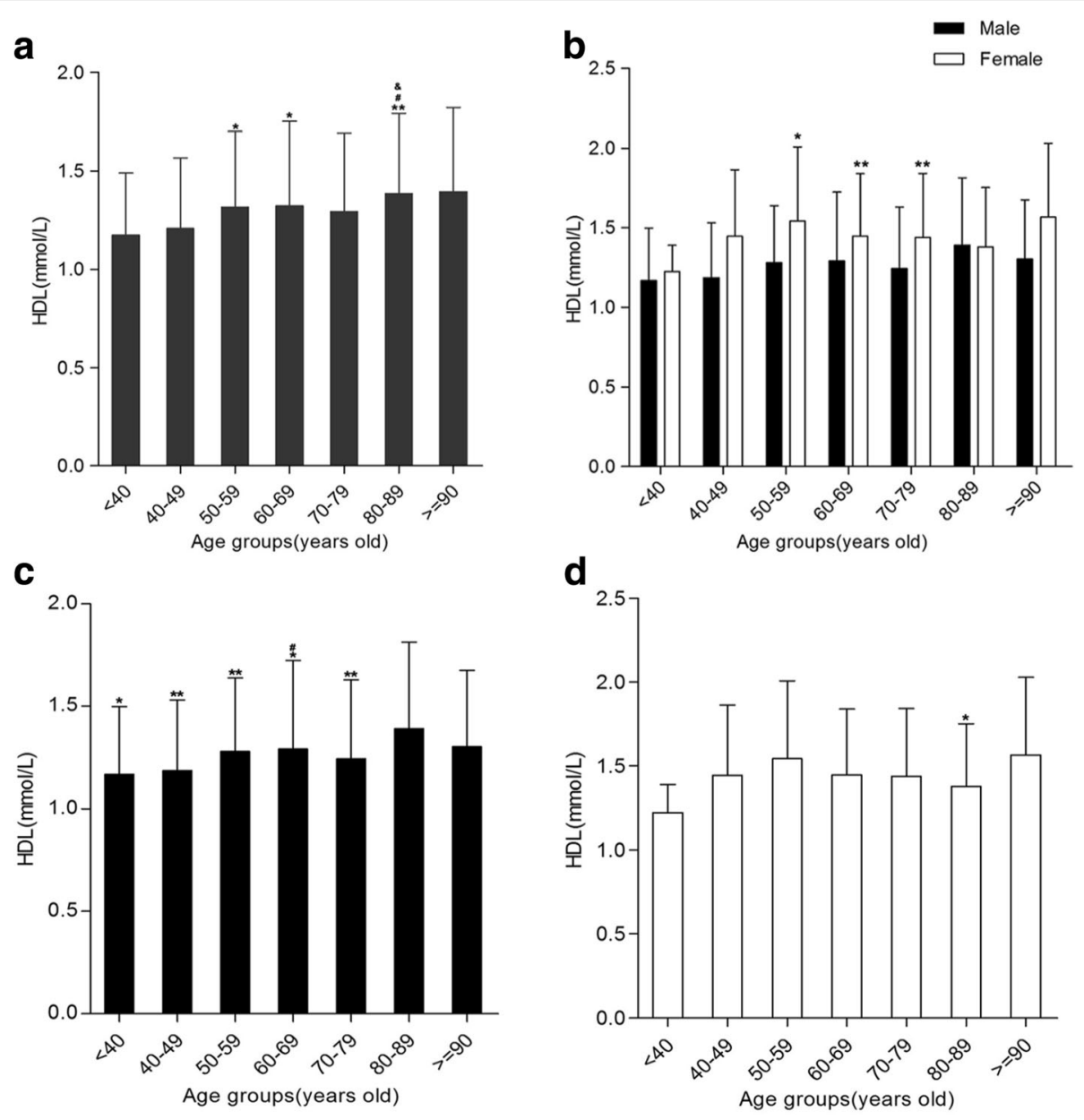

Fig. 3 High-density lipoprotein (HDL) levels in various age groups. a Comparison between 40 and 49 year of age group and 50-59,60-69,80-89 years of various age groups, respectively, ${ }^{*} P<.05,{ }^{* *} P<.01$. Comparison between $<40$ and $80-89$ year age groups were made, ${ }^{\#} P<.05$. Comparison between 70 and 79 year age groups and $80-89$ year age groups were made, ${ }^{\&} P<.05 . \mathbf{b}$ The proportions of acute myocardial infarction patients who had high-density lipoprotein (HDL) levels. c For males, comparison between 80 and 89 year of age group and less than 79 years of various age groups, respectively, ${ }^{*} P$ $<.05$, ${ }^{*} P<.01$. Comparison between 40 and 49 and $60-69$ year age groups were made, ${ }^{\#} P<.05$. $\mathbf{d}$ For females, comparison between 50 and 59 and $80-89$ year age group. ${ }^{*} P<.05$

higher for males. For the elderly, males had lower proportions of isolated high LDL, but proportion of isolated low HDL is higher than females. At the same time, the proportions of high TG were lower in the elderly than those in the nonelderly, while contrary to low HDL for males. Similarly, males had lower high LDL and high TG than females for elderly patients, while contrary to low HDL.

\section{Discussion}

Lipid abnormality is one of important risk factor for cardiovascular disease in AMI patients. In particular, LDL-C and HDL-C are important factor for atherosclerosis and cardiovascular disease development [1]. More and more evidences that a decrease in LDL-C levels or a increase in HDL levels can prevent the occurrence of cardiovascular disease [4]. This study was aimed at investigating serum lipid levels in AMI patients in southern China. Our research show that, although all enrolled patients were Hakka population (Han Chinese immigrated from northern China to southern China hundreds or thousand years ago) and came from the same geographical region (Meizhou, a most populated city with Hakka peoples in China), different age and gender subgroups had different lipid abnormality patterns.

Compared with the elderly, the nonelderly have higher levels of LDL for males. In addition, data indicated that prevalence of combined dyslipidemia was higher in nonelderly $(59.4-68.9 \%)$ than in elderly $(52-56.9 \%)$. It indicated that dyslipidemia was more prevalent among the nonelderly than the elderly. Therefore, it was necessary for the nonelderly to accepted lipid-lowering therapy and choose more effective lipid-lowering drugs. TG 

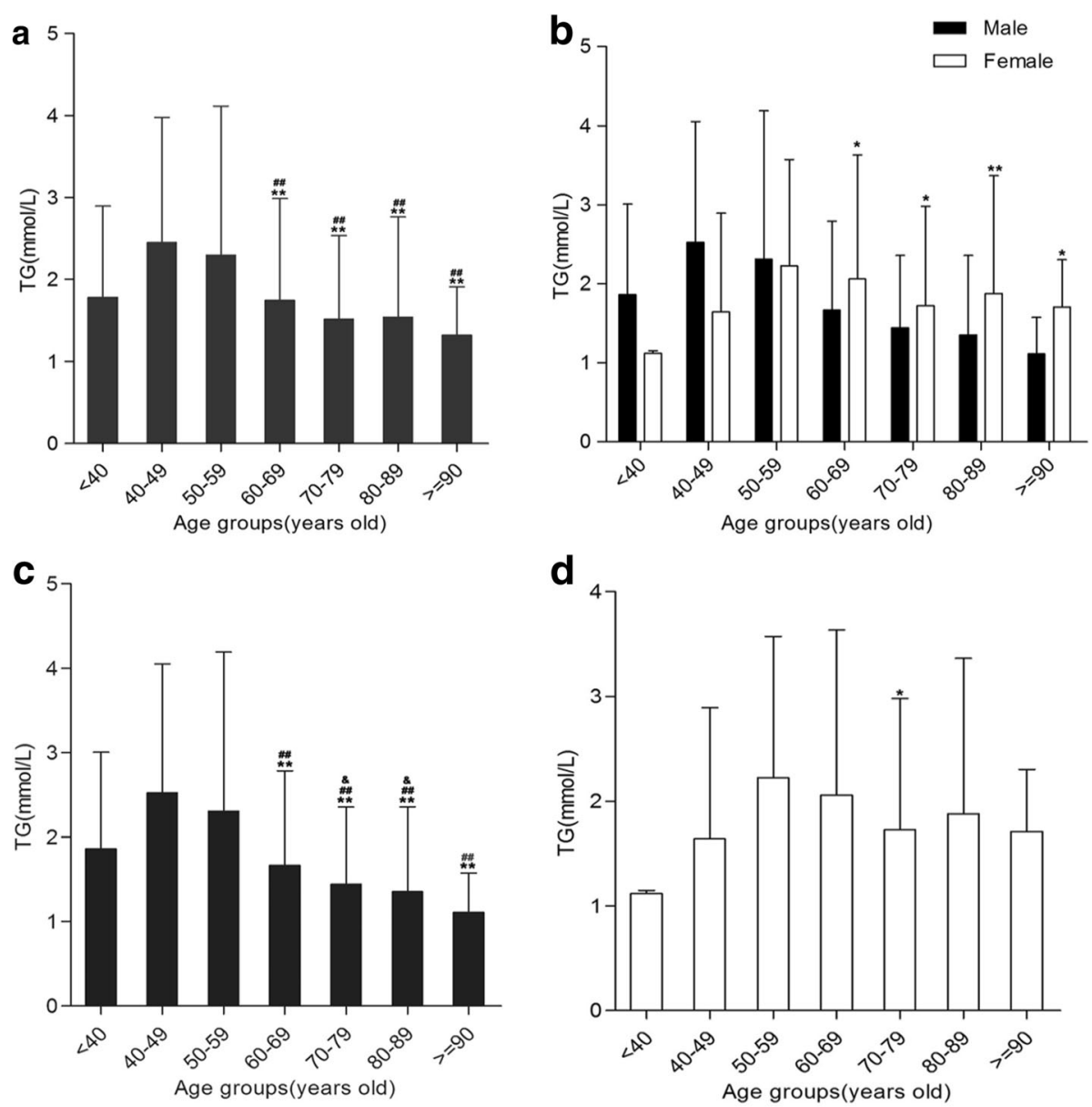

Fig. 4 Triglyceride (TG) levels in various age groups. a Crossing comparisons of arbitrary two age groups between $<40$, 40-49 year of age groups and more than 60 years of various age groups, respectively, ${ }^{*} P<.05,{ }^{*} P<.01,{ }^{*} P<.05,{ }^{\#} P<.01$. b Compared with males in the same age group, ${ }^{*} P$ $<.05,{ }^{*} P<.01$. c For males, Crossing comparisons of arbitrary two age groups between 40 and $49,50-59$ year of age groups and more than 60 years of various age groups, ${ }^{*} P<.05,{ }^{* *} P<.01,{ }^{\#} P<.05,{ }^{\# \#} P<.01$. Comparison between 60 and 69 and $70-79,80-89$ year age groups was made, ${ }^{\&} P<.05$. d For females, Comparison between 50 and 59 and $70-79$ year age groups was made, ${ }^{*} P<.05$

levels in nonelderly were higher compared to elderly for both males and females in south China. Compare with males, females had higher levels of TC and TG in both 50-59, 60-69 year age groups, respectively, which were considered as the perimenopausal window for women. This results was inconsistent with the conclusions of Drs Wei et al. [10]. Previous studies proved estrogen play an important role in lipid metabolism [11]. LDL, TC and TG of postmenopausal women were increased in China [12].There may be unique environmental and socioeconomic factors due to high TG levels in nonelderly with AMI. Changes in diet and lifestyle may be were main factors, which reflect in residents of the area prefer to eat greasy food that increases the risk of dyslipidemia. At the same time, several studies also have shown that elevated TG levels are strongly related to lifestyle factors, which including increased body mass index, lower physical activity levels, increased intake of high calorie foods, and the specific mechanism was unclear [13-15].

According to the diagnostic criteria of dyslipidemia from guidelines for the prevention of dyslipidemia in Chinese adults 2007, Our data indicated that 31.1-40.6\% of the nonelderly and $43.1-48 \%$ of the elderly AMI patients had normal lipids, and that AMI patients who had LDL levels $<80 \mathrm{mg} / \mathrm{dL}$ accounted for $23 \%$. Therefore, most of AMI patients require lipid-lowering therapy in the south of China. Studies had shown that early statin treatment reduced mortality, at the same time, it also can significantly improve the prognosis of patients with myocardial infarction who regardless of the level of blood lipids [16, 17]. The main mechanism is that statins are used to delay the progression of coronary atherosclerosis, minimize and stabilize plaques, eventually reducing 
a

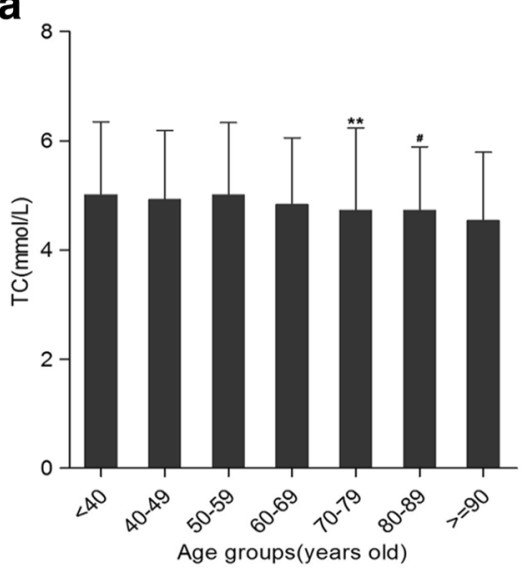

$\mathbf{C}_{8}$

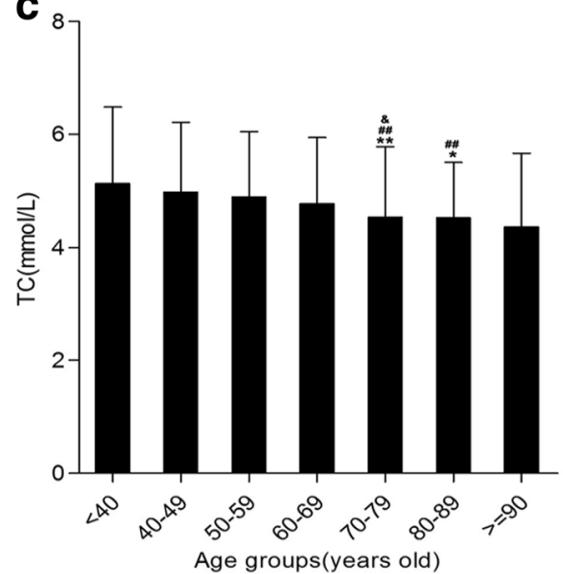

b

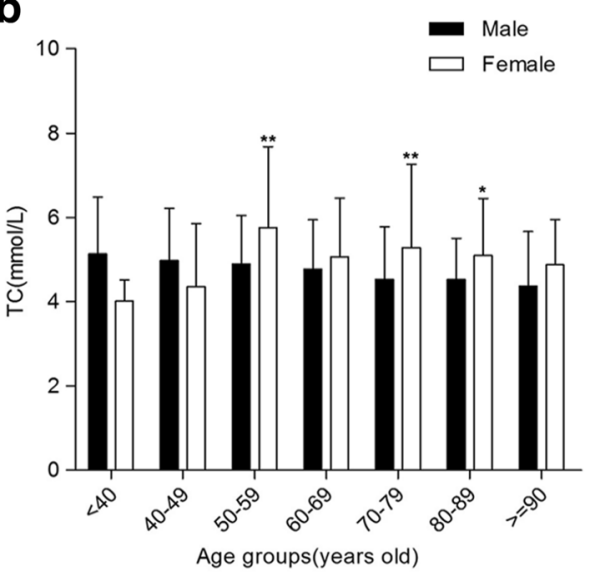

d

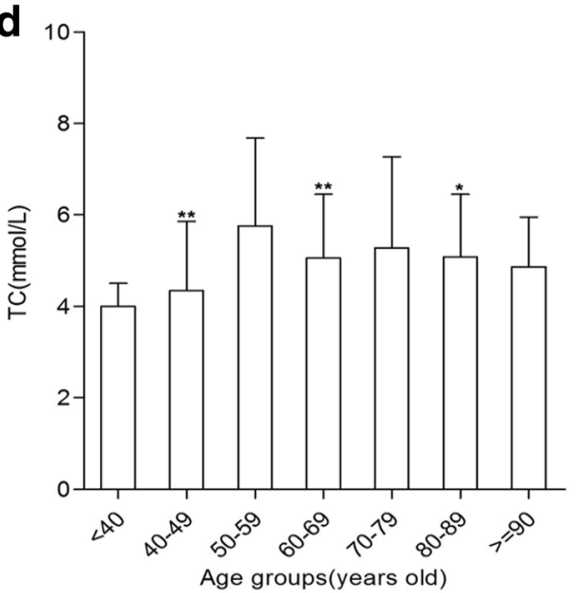

Fig. 5 Total cholesterol (TC) levels in various age groups. a Comparison between 50 and 59 year of age group and 70-79,80-89 years of various age groups, respectively, ${ }^{*} P<.05,{ }^{*} P<.01$. b Compared with males in the same age group, ${ }^{*} P<.05,{ }^{* *} P<.01$. c For males, Crossing comparisons of arbitrary 2 age groups between 40 and $49,50-59$ year of age groups and 70-79, 80-89 years of age groups, ${ }^{*} P<.05$, ${ }^{* *} P<.01 .{ }^{\#} P<.05,{ }^{\# \#} P<.01$. Comparison between 50 and 59 and 70-79 year age groups, ${ }^{\&} P<.05$. d For females, Comparison between 50 and 59 and 40-49,60-69,8089 year age group, respectively, $*<.05$, ${ }^{* *} P<.01$

Table 2 Combined dyslipidemia in Hakka patients with AMI

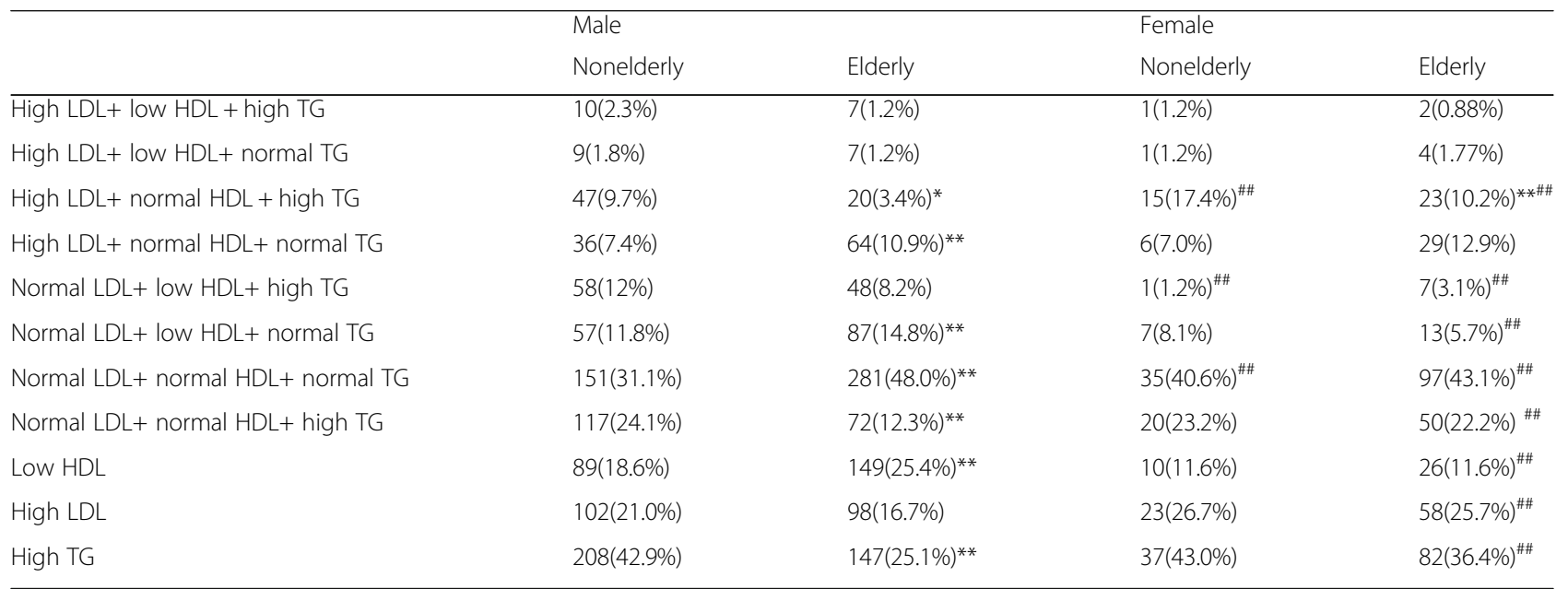

$\overline{A M I}$ acute myocardial infarction, $H D L$ high-density lipoprotein cholesterol, $L D L$ low-density lipoprotein cholesterol, $T G$ triglyceride Compared with the nonelderly of the same sex, ${ }^{*} P<.05$ and ${ }^{*} P<.01$

Compared with females of the same age group, ${ }^{\# \#} P<.01$ 
cardiovascular events. Thus, it is necessary promoted intensive lipid-lowering therapy for AMI patients who had normal lipid files [18].

Our study found that compared with females, males had higher LDL and TG for elderly patients, while contrary to low HDL. LDL-C and HDL-C were considered to produce adverse effects on the risk of myocardial infarction. The level of LDL can be reduced to the low limit approaching normal state $(<70 \mathrm{mg} / \mathrm{dL})$ by statin therapies [19]. However, statins do not completely prevent cardiovascular events, which data shown that cardiovascular events were reduced only $25-40 \%[20,21]$. In addition elevated LDL-C levels, lower level of HDL is strongly associated with cardiovascular events. Although there has been little acceptable treatment to improve HDL levels, these findings have important implications for lipid-lowering therapy in patients with acute myocardial infarction, in which lower HDL levels was associated with increased risk for myocardial infarction even if statin-treated patients who achieve LDL-C $<70 \mathrm{mg} / \mathrm{dL}$.

\section{Conclusion}

In conclusion, our results confirmed that serum lipid levels varied in age and sex in Hakka patients with acute myocardial infarction in southern China. Dyslipidemia is more prevalent in the non-elderly than elderly for males. Levels of TC, LDL, HDL, and TG were higher in females than males for the elderly Hakka population in southern China. Therefore, they may require lipid-lowering therapy to avoid the increased risk of cardiovascular disease. The most common combined dyslipidemia was the isolated high TG for AMI patients, and reduce TG may be critical to such patients.

\section{Abbreviations \\ AMI: Acute myocardial infarction; CAD: Coronary artery disease; \\ CHD: Coronary heart disease; CVD: Cardiovascular disease; \\ CVD: Cerebrovascular disease; HDL: High-density lipoprotein cholesterol; \\ LDL: Low-density lipoprotein cholesterol; TC: Total cholesterol; \\ TG: Triglyceride}

\section{Acknowledgments}

The authors would like to thank other colleagues whom were not listed in the authorship of Center for Cardiovascular Diseases, Clinical Core Laboratory and Center for Precision Medicine, Meizhou People's Hospital (Huangtang Hospital), Meizhou Hospital Affiliated to Sun Yat-sen University for their helpful comments on the manuscript. We would like to thank all of the participants in this study.

\section{Funding}

This study was supported by The National Key Research and Development Program of China (Grant No: 2016YFD0050405 to Dr. Pingsen Zhao), Natural Science Foundation of Guangdong Province, China (Grant No: 2014 A030307042 to Dr. Pingsen Zhao), Medical Scientific Research Foundation of Guangdong Province, China (Grant No.: A2016306 to Dr. Pingsen Zhao), Natural Science Foundation of Guangdong Province, China (Grant No.: 2016 A030307031 to Dr. Pingsen Zhao), The National Key Research and Development Program of China (Grant No.: 2017YFD0501705 to Dr. Pingsen Zhao), Key Scientific and Technological Project of Meizhou People's Hospital (Huangtang Hospital), Meizhou Hospital Affiliated to Sun Yat-sen University, Guangdong Province, China (Grant No:: MPHKSTP-20170102 to Pingsen Zhao) and Key Scientific and Technological Project of Meizhou People's Hospital (Huangtang Hospital),
Meizhou Hospital Affiliated to Sun Yat-sen University, Guangdong Province, China (Grant No.: MPHKSTP-20170101 to Zhixiong Zhong).

\section{Availability of data and materials}

Data are available from the authors on request.

\section{Authors' contributions}

$\mathrm{ZZ}$ and $\mathrm{JL}$ contributed equally to the work. PZ conceived and designed the study and manuscript revision. PZ and ZZ analyzed the data and wrote the manuscript. ZZ, BL, CL, ZL, MY and QZ collected and analyzed clinical data of the patients. $J L$ performed the laboratory testing. All authors read and approved the final manuscript.

Ethics approval and consent to participate

Not applicable.

\section{Consent for publication}

Not applicable.

\section{Competing interests}

The authors declare that they have no competing interests.

\section{Publisher's Note}

Springer Nature remains neutral with regard to jurisdictional claims in published maps and institutional affiliations.

\section{Author details}

${ }^{1}$ Center for Cardiovascular Diseases, Meizhou People's Hospital (Huangtang Hospital), Meizhou Hospital Affiliated to Sun Yat-sen University, Meizhou 514031, People's Republic of China. ${ }^{2}$ Clinical Core Laboratory, Meizhou People's Hospital (Huangtang Hospital), Meizhou Hospital Affiliated to Sun Yat-sen University, Meizhou 514031, People's Republic of China. ${ }^{3}$ Center for Precision Medicine, Meizhou People's Hospital (Huangtang Hospital), Meizhou Hospital Affiliated to Sun Yat-sen University, Meizhou 514031, People's Republic of China.

Received: 9 June 2017 Accepted: 5 December 2017

Published online: 16 December 2017

\section{References}

1. Mathers $C D$, Loncar D. Projections of global mortality and burden of disease from 2002 to 2030. PLoS Med. 2006;3:e442.

2. Zhang W, Ji F, Yu X, Wang X. Factors associated with unattained LDLcholesterol goals in Chinese patients with acute coronary syndrome one year after percutaneous coronary intervention. Medicine. 2017;96:e5469.

3. Lennep JERV. Risk factors for coronary heart disease: implications of gender. Cardiovasc Res. 2002:53:538.

4. Yong W, Qi B, Xu J, Zhou G, Chen S, Ping O, Liu S. Age- and sex-related difference in lipid profiles of patients hospitalized with acute myocardial infarction in East China. J Clin Lipidol. 2014;8:562-7.

5. Perk J, Backer GD, Gohlke H, Graham I, Reiner Ž, Verschuren WMM, Albus C, Benlian P, Boysen G, Cifkova R, Deaton C, Ebrahim S, Fisher M, Germano G, Hobbs R, Hoes A, Karadeniz S, Mezzani A, Prescott E, Ryden L, Scherer M, Syvänne M, Scholte Op Reimer WJM, Vrints C, Wood D, Zamorano JL, Zannad F. Developed with the special contribution of the European Association for Cardiovascular Prevention \& Rehabilitation (EACPR). European Guidelines on Cardiovascular Disease Prevention in Clinical Practice (Version 2012). Int J Behav Med. 2012:19:403-88.

6. Yusuf S, Hawken S, Ounpuu S, Dans T, Avezum A, Lanas F, Mcqueen M, Budaj A, Pais P, Varigos J. Effect of potentially modifiable risk factors associated with myocardial infarction in 52 countries (the INTERHEART study): case-control study. Orv Hetil. 2006;147:675.

7. Schenkein HA, Loos BG. Inflammatory mechanisms linking periodontal diseases to cardiovascular diseases. J Clin Periodontol. 2013;40:S51.

8. Xiang QL, Yong HZ, Wong MCS, Yan JX, Hao FX, Shao PN, Wen JM, Thomas GN, ITS Y. The prevalence of metabolic syndrome and cardiovascular risk factors in adults in southern China. BMC Public Health. 2012;12:64.

9. Prevention JCfDCgo, Adults ToDi. Chinese guidelines on prevention and treatment of dyslipidemia in adults. Zhonghua Xin Xue Guan Bing Za Zhi. 2007;35:390. 
10. Wei Y, Qi B, Xu J, Zhou G, Chen S, Ouyang P, Liu S. Age- and sex-related difference in lipid profiles of patients hospitalized with acute myocardial infarction in East China. J Clin Lipidol. 2014;8:562-7.

11. Wang F, Li J, Chu D. The changes of lipid levels in postmenopause female patients with coronary heart disease. Chin J Cardiol. 1999;27:102-4.

12. Group TECW. Hormones and cardiovascular health in women. Hum Reprod Update. 2006;12:483.

13. Miller M, Stone NJ, Ballantyne C, Bittner V, Criqui MH, Ginsberg HN, Goldberg AC, Howard WJ, Jacobson MS, Krisetherton PM. Triglycerides and cardiovascular disease: a scientific statement from the American Heart Association. Circulation. 2011;123:2292-333.

14. Tirosh A, Rudich A, Shochat T, Tekesmanova D, Israeli E, Henkin Y, Kochba I, Shai I. Changes in triglyceride levels and risk for coronary heart disease in young men. Ann Intern Med. 2007;147:377-85.

15. Durstine JL, Grandjean PW, Cox CA, Thompson PD. Lipids, lipoproteins, and exercise. J Cardpulm Rehabil. 2002;22:385-98.

16. Dohi T, Miyauchi K, Okazaki S, Yokoyama T, Yanagisawa N, Tamura H, Kojima T, Yokoyama K, Kurata T, Daida H. Early intensive statin treatment for six months improves long-term clinical outcomes in patients with acute coronary syndrome (extended-ESTABLISH trial): a follow-up study. Atherosclerosis. 2010;210:497-502.

17. Teshima Y, Yufu K, Akioka H, Iwao T, Anan F, Nakagawa M, Yonemochi $H$, Takahashi N, Hara M, Saikawa T. Early atorvastatin therapy improves cardiac function in patients with acute myocardial infarction. J Cardiol. 2009;53:58-64.

18. Brunzell JD, Davidson M, Furberg CD, Goldberg RB, Howard BV, Stein JH, Witztum JL. Lipoprotein management in patients with cardiometabolic risk: consensus statement from the American Diabetes Association and the American College of Cardiology Foundation. J Am Coll Cardiol. 2008:51:1512-24.

19. Ki Hong L, Myung Ho J, Ha Mi K, Youngkeun A, Jong Hyun K, Shung Chull C, Young Jo K, Seung Ho H, Whan S, Taek Jong H. Benefit of early statin therapy in patients with acute myocardial infarction who have extremely low low-density lipoprotein cholesterol. J Am Coll Cardiol. 2011;58:1664.

20. Feasson S. Primary prevention of cardiovascular disease with atorvastatin in type 2 diabetes in the collaborative atorvastatin diabetes study (CARDS) : multicentre randomised placebo-controlled trial, 685-696. J Mal Vasc. 2005;30:63-4.

21. Shepherd J, Betteridge J, Van GL, Panel EC. Nicotinic acid in the management of dyslipidaemia associated with diabetes and metabolic syndrome: a position paper developed by a European consensus panel. Curr Med Res Opin. 2005;21:665.

\section{Submit your next manuscript to BioMed Central and we will help you at every step:}

- We accept pre-submission inquiries

- Our selector tool helps you to find the most relevant journal

- We provide round the clock customer support

- Convenient online submission

- Thorough peer review

- Inclusion in PubMed and all major indexing services

- Maximum visibility for your research

Submit your manuscript at www.biomedcentral.com/submit

CBiomed Central 